\title{
The Effect of Tualang Honey in Chronic Exposure of High Cholesterol Diet in Animal Model
}

Roslina Abdul Rahim¹, Siti Aesah @ Naznin Muhammad², Azril Shahreez Abdul Ghani ${ }^{1}$, Nor Zamzila Abdullah², Norlelawati A. Talib², Norra Harun ${ }^{3}$

${ }^{1}$ Department of Basic Medical Science, Kulliyyah of Medicine, International Islamic University Malaysia

2Department of Pathology and Laboratory Medicine, Kulliyyah of Medicine, International Islamic University Malaysia

${ }^{3}$ Department of Pathology, Hospital Tengku Ampuan Afzan, Kuantan Pahang.

Introduction: Hyperlipidaemia accompanies chronic renal disease either as a consequence of the renal dysfunction or as part of generalized metabolic derangements. The aim of this study was to determine the effects of tualang honey (TH) on the kidneys of animal model with chronic exposure to high cholesterol diet. Materials and method: Twenty Sprague-Dawley rats were divided into two groups, the high cholesterol diet $(12 \%$ CD $(n=16)$ and standard diet $(S D)(n=4)$ and were fed for 12 weeks. After 12 weeks, the rats in the $12 \%$ CD group were subsequently divided into four groups. The first group was continued with only $12 \% \mathrm{CD}$ while the other 3 groups in addition to the $12 \% \mathrm{CD}$, they were given $\mathrm{TH}$ treatment at different concentrations (1.2, 2.4 and $3.0 \mathrm{~g} / \mathrm{kg} /$ day) for 4 weeks. Biochemical analysis of lipid profile and renal function were performed at the end of the experiment. The animals were sacrificed and the kidneys were harvested for histological examination. Results: In the 12 weeks HCD group, the serum cholesterol, LDL-C and creatinine levels were significantly higher) compared to that of the SD group. All groups with the tualang honey treatment had significant reduction in the LDL-C, triglyceride and creatinine levels. Histological examination of the kidney tissues of the HCD and $\mathrm{HCD}+\mathrm{TH}$ groups at 16 weeks revealed segmental mesangial proliferation of some glomeruli with focal mesangial matrix expansion. No areas of periglomerular and peritubular fibrosis were observed. Conclusion: Tualang honey supplementation of animal model with chronic exposure to high cholesterol diet improved the renal function hence suggesting the its renoprotective effect. However, there were no changes seen in the histology of the kidneys. Additionally, tualang honey showed improvement in the LDL-C and triglyceride levels indicating its lipid lowering activities. 